\title{
Automated Segmentation of the Liver from 3D CT Images Using Probabilistic Atlas and Multi-level Statistical Shape Model
}

\author{
Toshiyuki Okada ${ }^{1}$, Ryuji Shimada ${ }^{1}$, Yoshinobu Sato ${ }^{1}$, Masatoshi Hori ${ }^{2}$, Keita \\ Yokota $^{1}$, Masahiko Nakamoto ${ }^{1}$, Yen-Wei Chen ${ }^{3}$, Hironobu Nakamura ${ }^{2}$, \\ and Shinichi Tamura ${ }^{1}$ \\ ${ }^{1}$ Division of Image Analysis \\ 2 Department of Radiology, \\ Osaka University Graduate School of Medicine, Suita, Osaka, 565-0871, Japan \\ \{toshi, yoshi\}@image.med.osaka-u.ac.jp \\ ${ }^{3}$ College of Information Science and Engineering, Ritsumeikan University, Japan
}

\begin{abstract}
An atlas-based automated liver segmentation method from 3D CT images is described. The method utilizes two types of atlases, that is, the probabilistic atlas (PA) and statistical shape model (SSM). Voxel-based segmentation with PA is firstly performed to obtain a liver region, and then the obtained region is used as the initial region for subsequent SSM fitting to 3D CT images. To improve reconstruction accuracy especially for largely deformed livers, we utilize a multi-level SSM (ML-SSM). In ML-SSM, the whole shape is divided into patches, and principal component analysis is applied to each patches. To avoid the inconsistency among patches, we introduce a new constraint called the adhesiveness constraint for overlap regions among patches. In experiments, we demonstrate that segmentation accuracy improved by using the initial region obtained with PA and the introduced constraint for ML-SSM.
\end{abstract}

\section{Introduction}

Segmentation of the liver from 3D data is a prerequisite for computer-assisted diagnosis and preoperative planning. Prior information of the liver, typically represented as statistical atlases, is useful for robust segmentation. Two types of statistical atlases, a statistical shape model (SSM) [1] and a probabilistic atlas (PA) 2], have been utilized to increase robustness of the segmentation.

A SSM is widely used for organ segmentation and its potential performance for liver segmentation has been shown [3] 4]. However, previous methods using SSM [3] 4] had the following problems: (1)There is essential limitation on reconstruction accuracy especially for diseased livers involving large deformations and lesions. (2) Good initialization is required to obtain proper convergence. One approach to overcome the first problem is to use multi-level SSM (ML-SSM) [5], in which the whole organ shape is divided into multiple patches, which are further subdivided at finer representation levels. One problem of ML-SSM is, however,

N. Ayache, S. Ourselin, A. Maeder (Eds.): MICCAI 2007, Part I, LNCS 4791, pp. 86-93, 2007.

(C) Springer-Verlag Berlin Heidelberg 2007 
inconsistency among patches at finer levels. While previous work tried to solve the inconsistency problem [6], they did not apply the method to the liver, which has highly complex shape and large inter-patient variation. Another approach to address the first problem is to perform SSM fitting followed by shape constrained deformable model fitting [4. However, shape constraints inherent in the liver are not embedded in the deformable model, and robustness against large deformation and lesions has not been verified. Further, the second problem has not been addressed in the previous studies. Heimann et al. reported that not a few cases failed to converge due to the initialization problem [4].

An alternative approach to represent prior information is probabilistic atlas (PA) 2, where the existence probability of the liver is assigned to each voxel position. Recently, prediction accuracy of PA was shown to improve using spatial standardization based on surrounding structures [7] 8]. Zhou et al. showed that highly accurate segmentation was possible from non-contrasted CT images by using an automated method based on PA 8]. However, the datasets they used consisted of normal and a fraction of mildly diseased livers and did not verify the performance for severely diseased livers.

In this paper, we formulate an automated method for liver segmentation using both PA and ML-SSM. The features of our method are as follows: (1) Initialization for SSM fitting is automated using automated segmentation based on PA. (2) ML-SSM is used to improve reconstruction accuracy especially for largely deformed livers. (3) A new constraint is introduced to avoid inconsistency among patches at finer levels of ML-SSM. We experimentally evaluate the improvements of the performance by introducing the above features.

\section{Methods}

\subsection{Spatial Standardization Using the Abdominal Cavity}

Given training datasets, PA and ML-SSM are constructed. Before the construction, spatial standardization of the datasets is necessary. To do so, one CT dataset which was judged to have an average liver shape by a radiology specialist was selected as a standard patient, and its abdominal cavity is regarded as the standardized space to represent the standardized liver position and shape [7.

An individual patient dataset is mapped into the standardized patient space through nonrigid registration. We assume that the regions of the abdominal cavity and liver have already been manually segmented from 3D data. Let $A_{i}$ and $L_{i}$ denote the shapes of the $i$-th patient's abdominal cavity and liver, respectively, where $i=0,1,2, \ldots, n-1$. These shapes are represented by a $3 \mathrm{D}$ point dataset, which is the vertices of the decimated surface model generated from the manually segmented region of $3 \mathrm{D}$ data. Let $A_{0}$ be the abdominal cavity of the selected standard patient data. Let $T\left(\mathbf{x} ; A_{i}\right)$ be the dense $3 \mathrm{D}$ deformation vector field generated by nonrigid registration between $i$-th patient and the standard patient so that the abdominal cavity $A_{i}$ and $A_{0}$ are registered, where $\mathbf{x}$ is $3 \mathrm{D}$ position of the data. To generate the dense $3 \mathrm{D}$ deformation vector field, we use the point-based 


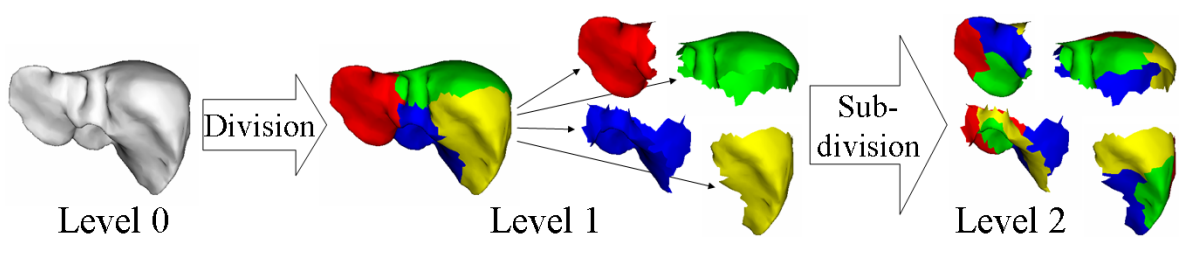

Fig. 1. Hierarchical division of the liver shape for multi-level statistical shape model

nonrigid registration method developed by Chui et al. 9]. The standardized liver shape $L_{i}^{\prime}$ of original shape $L_{i}$ is given by $L_{i}^{\prime}=\left\{\mathbf{x}+T\left(\mathbf{x} ; A_{i}\right) \mid \mathbf{x} \in L_{i}\right\}$.

\subsection{Constructing Statistical Atlases}

Constructing probabilistic atlas. Let $B_{i}(\mathbf{x})$ be a binary image where value 1 is assigned to inside of standardized liver $L_{i}^{\prime}$ and value 0 to other regions. The probabilistic atlas, $P(\mathbf{x})$, standardized using the abdominal cavity shape is defined as the average of $B_{i}(\mathbf{x})$ over $n$ patient datasets by $P(\mathbf{x})=(1 / n) \sum_{i=0}^{n-1} B_{i}(\mathbf{x})$.

Constructing multi-level statistical shape model. Let $L_{0}\left(=L_{0}^{\prime}\right)$ be the liver shape of the standard patient. Let $\mathbf{v}_{0 k}=\left(v_{0 k x}, v_{0 k y}, v_{0 k z}\right)(k=1, \cdots, m)$ denote the vertices of $L_{0}$, where $m$ is the number of the vertices. Let $\mathbf{q}_{0}$ denote the concatenation of $\mathbf{v}_{0 k}$, where $\mathbf{q}_{0}$ is a $3 m$-dimensional vector. Point-based nonrigid registration [9] between the standard liver $L_{0}$ and individual liver $L_{i}^{\prime}$ is performed to determine the correspondences of the vertices between the individual and standard livers.

To construct a multi-level surface model, $L_{i}$ is divided into $N_{1}$ sub-shapes (we call them "patches") [6]. These patches are recursively divided and multi-level surface model $L_{i \ell j}\left(j=1, \cdots, N_{\ell}\right)$ is constructed (Fig. 1), where $L_{i \ell j}$ denotes $j$-th patch at level $\ell$ of $i$-th patient, and $N_{\ell}$ denotes the number of patches at level $\ell\left(N_{0}=1\right)$. Each $L_{i \ell j}(i=0, \cdots, n-1)$ is normalized by its center of gravity. Let $\mathbf{q}_{i \ell j}$ denote concatenation of vertices of $L_{i \ell j}$. We assume that the adjacent patches at the same level overlap each other along their boundaries. These overlap regions are used to impose a constraint for eliminating inconsistency among patches, which will be described in the next subsection. From $n$ datasets of each patch, $\mathbf{q}_{i \ell j}(i=0, \cdots, n-1)$, the mean vector $\overline{\mathbf{q}}_{\ell j}$ is computed, and then principal component analysis (PCA) is applied for each patch independently to obtain eigenvectors $\Phi_{\ell j}$ corresponding to principal components. ML-SSM at level $\ell$ is defined as

$$
\mathbf{q}_{\ell j}\left(\mathbf{b}_{\ell j}\right)=\overline{\mathbf{q}}_{\ell j}+\Phi_{\ell j} \mathbf{b}_{\ell j} \quad\left(j=1, \cdots, N_{\ell}\right)
$$

where $\mathbf{b}_{\ell j}$ is the shape parameter vector at level $\ell$ of $j$-th patch.

\subsection{Segmentation of the Liver Using Statistical Atlases}

The proposed segmentation method using PA and ML-SSM consists of the following steps: 
1. Initial region extraction using voxel-based segmentation with PA [8].

2. Estimation of initial shape parameters by fitting SSM to the initial region.

3. Repeat the following multi-level segmentation processes until the finest level.

(a) Repeat the following segmentation processes for a fixed number of times.

i. Detection of edge points of the liver boundaries from CT data by searching along surface normals of current ML-SSM surface.

ii. Estimation of shape parameters by fitting ML-SSM to the edge points.

(b) Divide the current patches of ML-SSM into those at the next finer level.

In the following, details of step 1 , step 2, and segmentation processes at step $3(\mathrm{a})$ are described.

Initial region extraction using voxel-based segmentation with PA. Abdominal CT data is spatially standardized by the method described in section 2.1 (Fig. 2(a)], and smoothed with an anisotropic diffusion filter [3]. For the standardization, the abdominal cavity region is extracted automatically unlike the training phase (The extraction method is briefly described in 3.1). The volume of interest (VOI) is defined as the region where probabilistic atlas $P(\mathbf{x})($ Fig. 2(b) is larger than threshold value $T_{\text {map }}$. Let $I(\mathbf{x})$ be the standardized and smoothed image. Likelihood image $Q(\mathbf{x})$ is given by $Q(\mathbf{x})=\exp \left(-(I(\mathbf{x})-\bar{I})^{2} /\left(2 \sigma^{2}\right)\right)$, where $\bar{I}$ and $\sigma$ are average intensity and standard deviation, respectively, which are estimated based on histogram analysis inside the VOI [8]. $Q(\mathbf{x})$ is defined as the Gaussian of $I(\mathbf{x})$ and it is the largest when $I(\mathbf{x})$ is the same as the average intensity $\bar{I}$. Given $Q(\mathbf{x})$ and $P(\mathbf{x})$, combined likelihood image $Q^{\prime}(\mathbf{x})$ (Fig. 2(c) is defined as $Q^{\prime}(\mathbf{x})=Q(\mathbf{x}) P(\mathbf{x})$. Note that the voxel value of $Q^{\prime}(\mathbf{x})$ is normalized between 0 and 1 . The initial region is extracted by thresholding of $Q^{\prime}(\mathbf{x})$ using a fixed threshold value followed by opening and closing (Fig. $2(\mathrm{~d})$.

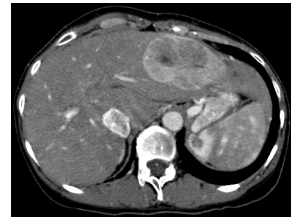

(a)

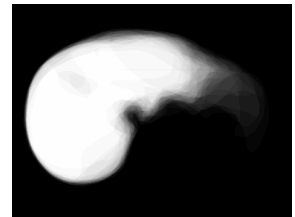

(b)

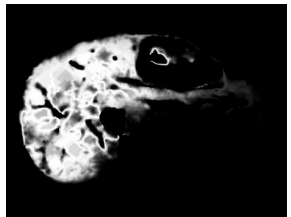

(c)

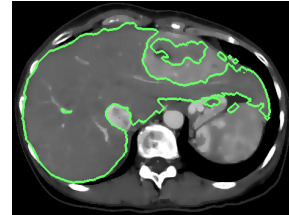

(d)

Fig. 2. Initial region segmentation processes using probabilistic atlas. (a) Standardized image. (b) Probabilistic atlas. (c) Combined likelihood image. (d) Extracted initial region (green contours).

Estimation of initial shape parameters. Let $R$ be the surface model generated from initial region. Given $R$, we obtain the initial shape parameter $\mathbf{b}_{0}$ by iteratively minimizing

$$
C_{D}\left(\mathbf{q}_{0}\left(\mathbf{b}_{0}\right) ; R\right)=\frac{1}{\left|\mathbf{q}_{0}\left(\mathbf{b}_{0}\right)\right|} \sum_{\mathbf{x} \in \mathbf{q}_{0}\left(\mathbf{b}_{0}\right)} w(d(\mathbf{x}, R)) d(\mathbf{x}, R)
$$




$$
+\frac{1}{|R|} \sum_{\mathbf{x} \in R} w\left(d\left(\mathbf{x}, \mathbf{q}_{0}\left(\mathbf{b}_{0}\right)\right)\right) d\left(\mathbf{x}, \mathbf{q}_{0}\left(\mathbf{b}_{0}\right)\right)
$$

where $d(\mathbf{x}, S)$ is the Euclidean distance between point $\mathbf{x}$ and surface $S$, and $|\cdot|$ denotes the number of vertices of surface model. Robust weight function $w(x)$ is used to deal with outliers due to large lesions [10. $w(x)$ is defined as $w(x)=\left\{\begin{array}{ll}1 & \text { if }|x| \leq s \\ s /|x| & \text { if }|x|>s\end{array}\right.$ in which $s$ is the robust standard deviation given by $s=\max \left(1.4826 \times \operatorname{median}\left\{\left|d_{k}-\operatorname{median}\left(d_{k}\right)\right|\right\}, 5.0 \mathrm{~mm}\right)$, and $d_{k}$ is residual in millimeters at each vertex.

Segmentation processes using ML-SSM. The edge points of the liver boundaries in CT images are detected from analysis of the CT value profile along the surface normal of the ML-SSM 3. In this study, the parameters needed for profile analysis are automatically determined using the result of histogram analysis obtained in the initial region extraction processes.

Detected edge points are fitted to ML-SSM. Let $P$ be the set of detected edge points from CT data. Let $\mathbf{q}_{\ell}$ and $\mathbf{b}_{\ell}$ denote concatenations of $\mathbf{q}_{\ell j}\left(\mathbf{b}_{\ell j}\right)$ and $\mathbf{b}_{\ell j}\left(j=1, \cdots, N_{\ell}\right)$, respectively. Given $P$ and level $\ell$, we estimate the shape parameters $\mathbf{b}_{\ell}$ by minimizing

$$
C\left(\mathbf{q}_{\ell}\left(\mathbf{b}_{\ell}\right) ; P\right)=C_{D}\left(\mathbf{q}_{\ell}\left(\mathbf{b}_{\ell}\right) ; P\right)+\lambda C_{A}\left(\mathbf{q}_{\ell}\right),
$$

where $C_{D}\left(\mathbf{q}_{\ell}\left(\mathbf{b}_{\ell}\right) ; P\right)$ is the sum of distances between model surface $\mathbf{q}_{\ell}$ and edge points $P$, and $C_{A}\left(\mathbf{q}_{\ell}\right)$ is the adhesiveness constraint for the overlap regions to eliminate the inconsistency among adjacent patches. Further, $\lambda$ is a weight parameter balancing the two constraints. $\lambda$ was determined experimentally. Letting $O_{\ell i}$ be the overlap regions, the cost functions of the adhesiveness constraint, $C_{A}\left(\mathbf{q}_{\ell}\right)$, is defined as

$$
C_{A}\left(\mathbf{q}_{\ell}\right)=\frac{1}{m_{\ell}} \sum_{i=1}^{N_{\ell}} \sum_{\mathbf{x} \in O_{\ell i}}\left(\mathbf{x}-\mathbf{x}^{\prime}\right)^{2},
$$

where $\mathbf{x}^{\prime}$ is the point that corresponds to $\mathbf{x}$ in the overlap region of the adjacent patch.

\section{$3 \quad$ Experimental Results}

\subsection{Experimental Conditions}

28 abdominal CT datasets (slice thickness $2.5 \mathrm{~mm}$, pitch $1.25 \mathrm{~mm}$, FOV $350 \times 350$ $\mathrm{mm}^{2}, 512 \times 512$ matrix, 159 slices) were used. We randomly selected 8 datasets for evaluation, and others for training. The probabilistic atlas and ML-SSM were constructed from the 20 training datasets. A radiology specialist judged the livers were largely deformed due to disease in 9 datasets among the 20 training datasets. Also, the livers were largely deformed in 5 datasets among the 8 evaluation datasets. 
Table 1. Evaluation results of segmentation accuracy. Averages of 8 datasets of Jaccard similarity measure and average distance [mm] (divided by slash) are shown in each experimental condition. The results of the proposed method are enhanced.

\begin{tabular}{|c|c|c|c|c|}
\hline Initialization & \multicolumn{2}{|l|}{ Using segmentation with PA } & \multicolumn{2}{c|}{ Using average shape } \\
\hline Adhesiveness constraint & $\lambda=0$ & $\lambda=0.2$ & $\lambda=0$ & $\lambda=0.2$ \\
\hline \hline Initial region & \multicolumn{2}{|c|}{$0.80 / 3.15$} & \multicolumn{2}{|c|}{$-/-$} \\
\hline Level 0 & \multicolumn{2}{|c|}{$0.80 / 3.20$} & \multicolumn{2}{|c|}{$0.79 / 3.61$} \\
\hline Level 1 & $0.84 / 2.36$ & $0.83 / 2.58$ & $0.84 / 2.60$ & $0.83 / 2.94$ \\
\hline Level 2 & $0.79 / 2.48$ & $\mathbf{0 . 8 6} / \mathbf{2 . 1 5}$ & $0.79 / 2.73$ & $0.85 / 2.54$ \\
\hline (Jaccard similarity measure / Average distance $[\mathrm{mm}])$.
\end{tabular}

While the abdominal cavity of the training data was manually segmented, that of the evaluation data was automatically extracted by combining the inner surface of the thoracic cage and the diaphragm surface approximated by a thinplate spline surface fitted to the lung bottom [8].

The number of SSM vertices at level 0 was 2500. The finest level of ML-SSM was 2 , that is, $\ell=0,1,2$. The numbers of patches were 4 at level 1 and 16 at level 2. The threshold value $T_{\text {map }}$ of probabilistic atlas $P(\mathbf{x})$ was set to 0.9 . The weight parameter $\lambda$ for the adhesiveness constraint was set to $\lambda=0.2$. The iteration count of segmentation processes at each level was 10 times.

For comparison purpose, the experiments were also performed under the following conditions. (1) The adhesiveness constraint was not used, that is, $\lambda=0$. (2) Instead of using the initial region obtained by voxel-based segmentation with PA, the average shape of the liver was used as the initial parameter of ML-SSM, that is, $\mathbf{b}_{0}=\mathbf{0}$.

To evaluate segmentation accuracy, we used two types of measures, Jaccard similarity measure [1] and average distance. The former is defined as $|A \cap B| / \mid A \cup$ $B \mid$, where $A$ and $B$ are the estimated region using ML-SSM and the corresponding manually segmented true region, respectively. The latter is the average of symmetric distances between the estimated and true surfaces [3].

\subsection{Results}

Table 1 summarizes the results of segmentation accuracy. When the weight parameter of the adhesiveness constraint was set to $\lambda=0.2$, segmentation accuracy was improved as hierarchy level of ML-SSM increased. When $\lambda=0.0$, however, segmentation accuracy was decreased at level 2 . The average distance of the proposed method using segmentation with PA as the initial region was smaller than using the average shape although there was not significant difference in Jaccard similarity measure. These results show the usefulness of using the adhesiveness constraint and segmentation with PA as the initial region, both of which have been introduced in this paper.

Figure 3 shows the result of the proposed method for each dataset $(\lambda=$ 0.2 , using initial segmentation with PA). In all evaluation datasets, ML-SSM at 

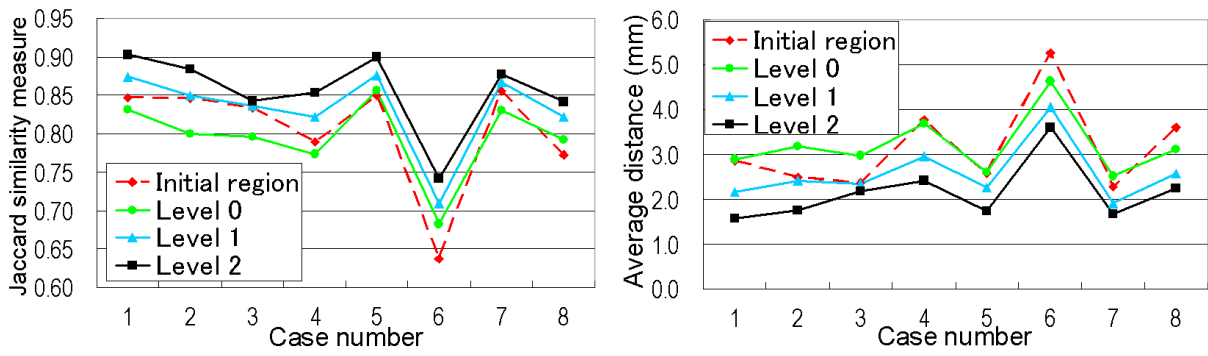

Fig. 3. Evaluation results of segmentation accuracy for each data by proposed method $(\lambda=0.2)$. Left: Jaccard similarity measure. Right: Average distance.

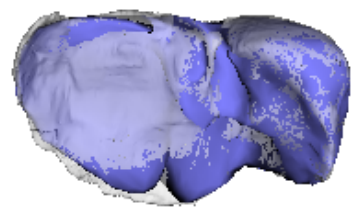

(a)

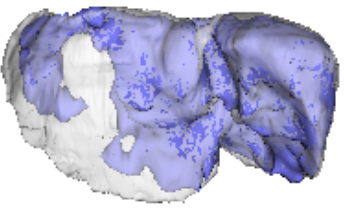

(b)

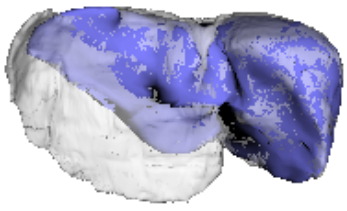

(c)

Fig. 4. Results of case 6. 3D surface models of the estimated shape (blue model) and the true shape (semitransparent white model) are superimposed so that their difference is easily understandable. (a) Region obtained by ML-SSM at level 2 using the proposed method. (b) Initial region obtained by voxel-based segmentation with PA. (c) Region obtained by ML-SSM at level 2 using average shape as initial region.

level 2 achieved the best results, and its Jaccard similarity measure and average distance were $0.86( \pm 0.05)$ and $2.15( \pm 0.62) \mathrm{mm}$ on average, respectively.

Figure 4 shows the results of case 6 , which had a large tumor (whose volume was more than $20 \%$ of the whole liver) and large deformation. The estimated liver shape by ML-SSM at level 2 using the proposed method (Fig. 4(a) was closer to the true shape than the initial region obtained by voxel-based segmentation with PA [8] (Fig. 4(b) and the result by ML-SSM at level 2 when the average shape was used as an initial region (Fig. 4(c).

\section{Discussion and Conclusions}

We have developed an automated segmentation method of the liver using statistical atlases. The proposed method was shown to improve segmentation accuracy for datasets including largely deformed livers by combining initial segmentation based on PA and subsequent ML-SSM fitting. We consider that the initial segmentation could capture boundary information even in deformed livers although not a few outlier boundaries were included. Subsequent ML-SSM fitting could deal with outlier boundaries to some extent, and further the edge detection accuracy was improved by hierarchization. Thus, the segmentation accuracy 
improved. We also demonstrate that the adhesiveness constraint was effective to deal with inconsistency at finer levels of ML-SSM. The accuracy was significantly degraded at the fine level (level 2) without the constraint.

In the current version of our method, automated extraction of the abdominal cavity is necessary for standardization of datasets to construct statistical atlases. Its extraction accuracy sometimes is insufficient. However, a circumscribed trapezoid of the thoracic cage, which is extracted in a highly stable manner [8, can be used for standardization instead of the abdominal cavity at the expense of slight degradation of the prediction accuracy of the constructed PA. As future work, we will evaluate the proposed segmentation approaches using different standardization methods including the above method. Further, we will evaluate the proposed approach by leave-one-out cross validation using large CT datasets.

\section{References}

1. Cootes, T.F., et al.: Active shape models - their training and application. Computer Vision and Image Understanding 61(1), 38-59 (1995)

2. Park, H., et al.: Construction of an abdominal probabilistic atlas and its application in segmentation. IEEE Trans. Med. Imaging 22(4), 483-492 (2003)

3. Lamecker, H., et al.: Segmentation of the liver using a 3D statistical shape model. Technical report, Zuse Institue, Berlin (2004)

4. Heimann, T., et al.: Active shape models for a fully automated 3D segmentation of the liver - an evaluation on clinical data. In: Larsen, R., Nielsen, M., Sporring, J. (eds.) MICCAI 2006. LNCS, vol. 4191, pp. 41-48. Springer, Heidelberg (2006)

5. Davatzikos, C., et al.: Hierarchical active shape models, using the wavelet transform. IEEE Trans. Med. Imaging 22(3), 414-423 (2003)

6. Zhao, Z., et al.: A novel 3D partitioned active shape model for segmentation of brain MR images. In: Duncan, J.S., Gerig, G. (eds.) MICCAI 2005. LNCS, vol. 3749, pp. 221-228. Springer, Heidelberg (2005)

7. Yokota, K., et al.: Construction of conditional statistical atlases of the liver based on spatial normalization using surrounding structures. In: Proceedings of Computer Assisted Radiology and Surgery (CARS), Osaka, Japan, pp. 39-40 (July 2006)

8. Zhou, X., et al.: Constructing a probabilistic model for automated liver region segmentation using non-contrast x-ray torso CT images. In: Larsen, R., Nielsen, M., Sporring, J. (eds.) MICCAI 2006. LNCS, vol. 4191, pp. 856-863. Springer, Heidelberg (2006)

9. Chui, H., et al.: A new point matching algorithm for non-rigid registration. Computer Vision and Image Understanding 89, 114-141 (2003)

10. Besl, P.J., et al.: Robust window operators. Machine Vision and Applications 2(4), 179-191 (1989)

11. Jaccard, P.: The distribution of the flora in the alpine zone. New Phytologist 11(2), 37-50 (1912) 